\title{
Sato's hyperfunctions and boundary values of monogenic functions
}

\author{
Irene Sabadini \\ Politecnico di Milano \\ Dipartimento di Matematica \\ Via Bonardi, 9 \\ 20133 Milano, Italy \\ irene.sabadini@polimi.it
}

\author{
Franciscus Sommen \\ Clifford Research Group \\ Faculty of Sciences \\ Ghent University \\ Galglaan 2, 9000 Gent, Belgium \\ Frank.Sommen@UGent.be
}

\author{
Daniele C. Struppa \\ Schmid College of Science and Technology \\ Chapman University \\ Orange 92866, CA, US \\ struppa@chapman.edu
}

\begin{abstract}
We describe the relationship between Sato's hyperfunctions and other theories of boundary values. In particular we show how monogenic functions can be used to represent classical hyperfunctions.
\end{abstract}

Key words: Boundary values; hyperfunctions; monogenic functions.

Mathematical Review Classification numbers: 30G35, 46F20.

Dedicated to Prof. Klaus Gürlebeck on the occasion of his 60th birthday

\section{Introduction}

Hyperfunctions are generalized functions introduced in the late 1950s by M. Sato, [22], [24] and can be thought of as the analytic equivalent of Schwartz's distributions, [26]. Indeed Sato was inspired by a belief that the natural setting for the analysis of singularities of solutions of differential equations was the analytic setting, rather than the differential one; for this reason his hyperfunctions must be constructed on real analytic manifolds, unlike distributions that only require a differentiable structure. For the sake of simplicity, one could confine the attention to the case of distributions and hyperfunctions defined on open sets in $n$-dimensional real Euclidean spaces, and since $\mathbb{R}^{n}$ is both differentiable and real analytic, both theories can be described in this setting. The original theory of Sato, in one variable, is quite intuitive and allows us to think of hyperfunctions as the difference of the boundary values of a holomorphic function across a singularity: we will discuss some examples later on. The generalization to the case of $n>1$ variables, however, is quite complex and relies on a different interpretation of hyperfunctions as elements in suitable relative cohomology groups. As such, a fully developed theory of hyperfunctions in several 
variables requires instruments from sheaf theory, category theory, and derived functors. This is clearly beyond the scope of this article, but despite this technical apparatus, it is still possible to offer an interpretation of hyperfunctions as (sums of) boundary values of suitable holomorphic functions. A very useful feature of hyperfunctions is that they form a flabby sheaf. Flabbiness allows to extend solutions of a differential equation globally, no matter what the singularities which may occur at the boundary of their original domain. This is an advantage with respect of working with distributions which may be not extendable beyond some points. One should note that distributions form a subsheaf of the sheaf of hyperfunctions.

One could wonder whether it may be possible to develop a theory of boundary values for functions that generalize holomorphic functions. This is indeed possible in several hyper complex contexts. For example, a full theory can be developed for boundary values of Cauchy-Fueter functions (see e.g. [10], [23], and most importantly [4]). In this paper, however, we will confine our attention to the case of boundary values of monogenic functions as introduced in [27] and [28].

In particular, in this paper we intend to revise some basic facts about monogenic hyperfunctions. These are a generalization of the now classical notion of hyperfunctions.

We also show that Clifford analysis allows to introduce monogenic hyperfunctions which are related to Sato's hyperfunctions and which lead to a simpler representation of Sato's hyperfunctions as boundary values.

Problems involving boundary values, though in a different setting have been treated in $[11,13,12]$.

The plan of the paper is the following: in Section 2 we recall the basic facts on Sato's hyperfunctions. In Section 3 we introduce monogenic hyperfunctions; for the sake of completeness and in order to illustrate the main ideas underlying the theory, we have added the proofs of some old results. Then we discuss some examples related to the Dirac delta on $\mathbb{R}^{n}$ and to the Riesz potential. Finally, in Section 4 we sketch some possible directions of research.

\section{Sato's hyperfunctions}

We provide here some basic material on this theory, and we refer the interested reader to [14], [15], [18] for all the proofs.

Let us consider an open set $\Omega \subset \mathbb{R}$; an open set $U \subset \mathbb{C}$ such that $\Omega$ is a closed subset of $U$, is said to be a complex neighborhood of $\Omega$.

Let us consider the complex vector space $\mathcal{O}(U \backslash \Omega)$ and its subspace $\mathcal{O}(U)$ where $\mathcal{O}$ denotes the sheaf of holomorphic functions.

Definition 2.1. Let $\Omega$ be an open set in $\mathbb{R}$. The vector space of hyperfunctions on $\Omega$ is defined as

$$
\mathcal{B}(\Omega)=\frac{\mathcal{O}(U \backslash \Omega)}{\mathcal{O}(U)},
$$

where $U$ is any complex neighborhoods of $\Omega$.

Thus a hyperfunction $f(x)$ as an equivalence class $f(x)=[F(z)]$ in this quotient. Any function $F(z)$ in the equivalence class is said to be a defining function for $f(x)$. 
The quotient $\mathcal{O}(U \backslash \Omega) / \mathcal{O}(U)$ depends a priori on the choice of the open set $U \subseteq \mathbb{C}$, but the next proposition, which relies on the Mittag-Leffler theorem, shows that this is not the case.

Proposition 2.2. Let $U$ and $U^{\prime}$ be two complex neighborhoods of the same open set $\Omega \subseteq \mathbb{R}$. Then, there is a vector space isomorphism

$$
\frac{\mathcal{O}(U \backslash \Omega)}{\mathcal{O}(U)} \cong \frac{\mathcal{O}\left(U^{\prime} \backslash \Omega\right)}{\mathcal{O}\left(U^{\prime}\right)}
$$

Example 2.3. Let $F \in \mathcal{O}(U \backslash \Omega)$ and denote by $f=[F]$ the hyperfunction $f$ defined by the quotient (1). If the function $F$ is holomorphic at every point of $\Omega$, then $f$ is the zero hyperfunction.

It is important to note that it is not possible to speak about the value of a hyperfunction at a given point, so it is not correct to think of $f(x)=0$ as a numerical value.

It is possible to give an important alternative definition of $\mathcal{B}(\Omega)$. Indeed, the relative cohomology sequence of the pair $(U, U \backslash \Omega)$ with coefficients in the sheaf $\mathcal{O}$ gives (with standard use of notations)

$$
\begin{gathered}
0 \rightarrow H_{\Omega}^{0}(U, \mathcal{O}) \rightarrow H^{0}(U, \mathcal{O}) \rightarrow H^{0}(U \backslash \Omega, \mathcal{O}) \\
\rightarrow H_{\Omega}^{1}(U, \mathcal{O}) \rightarrow H^{1}(U, \mathcal{O}) \rightarrow \ldots
\end{gathered}
$$

The Mittag-Leffler theorem implies that $H^{1}(U, \mathcal{O})=0$ and the unique continuation property for holomorphic functions gives $H_{\Omega}^{0}(U, \mathcal{O})=0$. By the above exact sequence we obtain

$$
\mathcal{B}(\Omega)=\frac{\mathcal{O}(U \backslash \Omega)}{\mathcal{O}(U)} \cong H_{\Omega}^{1}(U, \mathcal{O})
$$

The support of a hyperfunction $f \in \mathcal{B}(\Omega)$ is defined as the complement in $\Omega$ of the largest open subset on which $f$ vanishes, and we denote by $\mathcal{B}[K]$ the space of hyperfunctions supported by the compact set $K$. We have:

Theorem 2.4. Let $K$ be a compact set in $\mathbb{R}$ and let $U$ be any complex neighborhood of $K$. We have the following isomorphism

$$
\mathcal{B}[K] \cong \frac{\mathcal{O}(U \backslash K)}{\mathcal{O}(U)} .
$$

Note that, since a hyperfunction is determined by the equivalence class of a function $F \in \mathcal{O}(U \backslash \Omega)$, we can set $U \backslash \Omega=U^{+} \cup U^{-}$with $U^{ \pm}=U \cap\{ \pm \operatorname{Im} z>0\}$ and $F=\left(F^{+}, F^{-}\right)$, $F^{ \pm} \in \mathcal{O}\left(U^{ \pm}\right)$so that the hyperfunction $f$ can be represented by the pair $\left(F^{+}, F^{-}\right)$.

Consider the following hyperfunctions:

$$
\varepsilon(z)=\left\{\begin{array}{l}
1 \text { for } \operatorname{Im} z>0 \\
0 \text { for } \operatorname{Im} z<0
\end{array} \quad \bar{\varepsilon}(z)=\left\{\begin{array}{c}
0 \text { for } \operatorname{Im} z>0 \\
-1 \text { for } \operatorname{Im} z<0
\end{array}\right.\right.
$$

The hyperfunction $[\varepsilon]$ is defined on $\mathbb{R}$. It can be seen as the unit hyperfunction 1 if we look at it as the difference of boundary values of holomorphic functions. Obviously we have $[\varepsilon]=[\bar{\varepsilon}]$. This hyperfunction is used to construct a hyperfunction starting from a real analytic function $a(x)$ : it is sufficient to multiply $a(x)$ by the unit hyperfunction 1 . 
Example 2.5. (Boundary values) We now explain how a holomorphic function $F$ on $U \backslash \Omega$ defines a hyperfunction $f=[F]$ that can be seen as the boundary value of $F$. In fact, let us set

$$
F(x+i 0)=[F \cdot \varepsilon], \quad F(x-i 0)=[F \cdot \bar{\varepsilon}] .
$$

We have

$$
f(x)=F(x+i 0)-F(x-i 0)
$$

which gives formal meaning to the notion of boundary value.

This last example is important as it illustrates that there are hyperfunctions that are not distributions. In fact it is well known that all the distributions on the real line are in fact boundary values of holomorphic functions. However not all the boundary values of holomorphic functions are distributions, as explained in the next result, essentially due to Köthe, [16], and which we reformulate in the language (non-existent at the time) of hyperfunctions.

Theorem 2.6. Let $\Omega$ be an open set in $\mathbb{R}$ and let $U$ be a complex neighborhood of $\Omega$. Let $f=[F] \in \mathcal{B}(\Omega)$. The hyperfunction $f$ is a distribution on $\Omega$ if and only if for any compact set $K$ in $U$ there are positive constants $C, \eta$ and $N \in \mathbb{N}$ such that, if $0<|y|<\eta$, then

$$
\sup _{x \in K}|F(x+i y)| \leq C|y|^{-N} .
$$

Moreover, a distribution supported at the origin can be expressed as a finite linear combination of derivatives of the Dirac delta. It is not anymore the case with hyperfunctions, in view of the following

Proposition 2.7. Let $f \in \mathcal{B}(\{0\})$. Then

$$
f(x)=\sum_{j=0}^{+\infty} a_{j} \frac{d^{j}}{d x^{j}} \delta(x)
$$

where $\lim _{j \rightarrow+\infty} \sqrt[j]{j ! a_{j}}=0$.

Denoting by $\mathcal{A}, \mathcal{D}^{\prime}$ the sheaves of real analytic functions and of distributions on $\mathbb{R}$, we deduce the following proper inclusions:

$$
\mathcal{A} \subset \mathcal{D}^{\prime} \subset \mathcal{B}
$$

An important feature of the sheaf of hyperfunctions in contained in next result. Before to state it, we need the following definition:

Definition 2.8. A sheaf $\mathcal{F}$ on $\mathbb{R}^{n}$ is said to be flabby if for every open set $U \subset \mathbb{R}^{n}$ the restriction map $\mathcal{F}\left(\mathbb{R}^{n}\right) \rightarrow \mathcal{F}(U)$ is surjective.

Theorem 2.9. Let $\Omega \subseteq \mathbb{R}$. Then the correspondence

$$
\Omega \rightarrow \mathcal{B}(\Omega),
$$

defines a flabby sheaf $\mathcal{B}$ on $\mathbb{R}$. 
This is not the appropriate setting for a discussion of the notion of flabbiness, but we will simply note that this concept is of central importance in the study of sheaves of solutions of systems of differential equations.

Let us now move to hyperfunctions on $\mathbb{R}^{n}$. The definition requires more sophisticated tools and we will not discuss the details here but we limit ourselves to give the following:

Definition 2.10. Let $\Omega$ be an open set in $\mathbb{R}^{n}$ and let $U$ be any complex neighborhood of $\Omega$. Then

$$
\mathcal{B}(\Omega)=H^{n}(U, U \backslash \Omega ; \mathcal{O})=H^{n}\left(\mathbb{C}^{n}, \mathbb{C}^{n} \backslash \Omega ; \mathcal{O}\right)=H_{\Omega}^{n}\left(\mathbb{C}^{n}, \mathcal{O}\right) .
$$

It is possible to interpret hyperfunctions as boundary values but in a more complicated way, which involves the notion of infinitesimal wedge. Given an open set $\Omega \subseteq \mathbb{R}^{n}$, let $\mathbb{R}_{y}^{n}$ be the space of purely imaginary coordinates and let $\Gamma \subseteq \mathbb{R}_{y}^{n}$ be an open cone with vertex in the origin. A set of the form $\mathbb{R}^{n}+i \Gamma$ is called a wedge. A complex open set $U \subset \mathbb{C}^{n}$ is called an infinitesimal wedge of type $\Omega+i \Gamma 0$ if $U \subset \Omega+i \Gamma$ and for every proper subcone $\Gamma^{\prime}$ of $\Gamma$ and for every $\varepsilon>0$ there is $\delta>0$ such that

$$
U \supset \Omega_{\varepsilon}+i\left(\Gamma^{\prime} \cap\{|y|<\delta\}\right)
$$

where we have set $\Omega_{\varepsilon}=\{x \in \Omega: \operatorname{dist}(x, \partial \Omega)>\varepsilon\}$.

Definition 2.11. (Boundary value representation of hyperfunctions). Let $\left\{F_{\ell}(z)\right\}_{\ell=1, \ldots, m}$ be a set of holomorphic functions defined on infinitesimal wedges $\Omega+i \Gamma_{\ell} 0, \ell=1, \ldots, m$. The formal sum

$$
f(x)=\sum_{\ell=1}^{m} F_{\ell}\left(x+i \Gamma_{\ell} 0\right)
$$

is a hyperfunction on $\Omega$. result:

Hyperfunctions can be interpreted also as analytic functionals in the sense of the next

Theorem 2.12. Let $K$ be a compact set in $\mathbb{R}^{n}, n \geq 1$. The space $\mathcal{B}[K]$ of hyperfunctions with support contained in $K$ is isomorphic to $H_{K}^{n}\left(\mathbb{C}^{n}, \mathcal{O}\right)$. Moreover we have

$$
\mathcal{B}[K] \cong \mathcal{A}^{\prime}(K) \text {. }
$$

This quick introduction shows some main features of hyperfunctions that we will further discuss in the next section.

\section{Monogenic hyperfunctions}

By $\mathbb{C}_{n}$ we denote the real Clifford algebra over $n$ imaginary units $e_{1}, \ldots, e_{n}$ satisfying the relations $e_{i} e_{j}+e_{j} e_{i}=-2 \delta_{i j}$. An element in the Clifford algebra will be denoted by $\sum_{A} e_{A} x_{A}$ where $A=i_{1} \ldots i_{r}, i_{\ell} \in\{1,2, \ldots, n\}, i_{1}<\ldots<i_{r}$, is a multi-index, $e_{A}=e_{i_{1}} e_{i_{2}} \ldots e_{i_{r}}$ and $e_{\emptyset}=1$. The norm of an element $\sum_{A} e_{A} x_{A} \in \mathbb{C}_{n}$ is defined by $|x|^{2}=\sum_{A}\left|x_{A}\right|^{2}$. As it is well known, $\mathbb{C}_{1}$ contains bicomplex numbers, while $\mathbb{C}_{2}$ is the set of biquaternions $\mathbb{H} \otimes \mathbb{H}$.

An element $\left(x_{0}, x_{1}, \ldots, x_{n}\right) \in \mathbb{R}^{n+1}$ will be identified with the element $x=x_{0}+\underline{x}$ called, in short, paravector. A function $f: U \subseteq \mathbb{R}^{n+1} \rightarrow \mathbb{C}_{n}$ is seen as a function $f(x)$ of the paravector $x$. 
Definition 3.1. Let $\Omega \subseteq \mathbb{R}^{n+1}$ be an open set. Then $\mathcal{M}_{l}(\Omega)$ (resp. $\mathcal{M}_{r}(\Omega)$ ) denotes the right $\mathbb{C}_{n}$-module of real differentiable functions satisfying

$$
D f=\left(\frac{\partial}{\partial x_{0}}+\sum_{j=1}^{n} e_{j} \frac{\partial}{\partial x_{j}}\right) f=0 \quad\left(\text { resp. } f D=\frac{\partial}{\partial x_{0}} f+\left(\sum_{j=1}^{n} \frac{\partial}{\partial x_{j}} f e_{j}\right)=0\right) .
$$

As it is well known $D$ is called generalized Cauchy-Riemann operator and the functions in its kernel are said to be monogenic. The $\mathbb{C}_{n}$-modules $\mathcal{M}_{l}(\Omega)\left(\right.$ resp. $\left.\mathcal{M}_{r}(\Omega)\right)$ endowed with the topology of the uniform convergence on the compact subsets of $\Omega$ are Fréchet modules, see [27].

Let $\Omega \subseteq \mathbb{R}^{n}$ be an open set and let $U \subseteq \mathbb{R}^{n+1}$ be an open set such that $\Omega$ is relatively closedin $U$.

Definition 3.2. The set of left monogenic hyperfunctions in $\Omega$ is defined as

$$
\mathcal{B}_{l}(\Omega)=\frac{\mathcal{M}_{l}(U \backslash \Omega)}{\mathcal{M}_{l}(U)} .
$$

The fact that the definition is well posed and does not depend on the choice of the open set $U$ is based on the validity of the Mittag-Leffler theorem, see [2, Theorem 21.2].

Theorem 3.3. (Mittag-Leffler) Let $\mathcal{U}=\left\{U_{i}\right\}_{i \in I}$ be a locally finite open covering of $U$ and let $g_{i j} \in \mathcal{M}_{l}\left(U_{i} \cap U_{j}\right)$, with $U_{i} \cap U_{j} \neq \emptyset$ be such that

$$
g_{i j}+g_{j i}=0
$$

and if $U_{i} \cap U_{j} \cap U_{k} \neq \emptyset$

$$
g_{i j}+g_{j k}+g_{k i}=0 .
$$

Then there exist function $g_{j} \in \mathcal{M}_{l}\left(U_{i}\right)$ such that

$$
g_{j k}=g_{k}-g_{j} \quad \text { in } U_{j} \cap U_{k} .
$$

We now show that Definition 3.2 is well posed by proving the following fact, which is well known in the case of Sato's hyperfunctions:

Proposition 3.4. Let $U, U^{\prime}$ be two open sets in $\mathbb{R}^{n+1}$ such that $\Omega$ is relatively closed in $U$ and in $U^{\prime}$. Then

$$
\frac{\mathcal{M}_{l}(U \backslash \Omega)}{\mathcal{M}_{l}(U)} \cong \frac{\mathcal{M}_{l}\left(U^{\prime} \backslash \Omega\right)}{\mathcal{M}_{l}\left(U^{\prime}\right)}
$$

Proof. It is not reductive to assume that $U^{\prime} \subset U$. Consider the restriction map

$$
\rho: \frac{\mathcal{M}_{l}(U \backslash \Omega)}{\mathcal{M}_{l}(U)} \rightarrow \frac{\mathcal{M}_{l}\left(U^{\prime} \backslash \Omega\right)}{\mathcal{M}_{l}\left(U^{\prime}\right)} .
$$

The homomorphism $\rho$ is obviously injective since two monogenic functions $f$ and $g$ that coincide on $U^{\prime} \backslash \Omega$ must coincide on $U \backslash \Omega$. Thus we have to show that $\rho$ is also surjective. The Mittag-Leffler theorem asserts that if $V$ and $V^{\prime}$ are two open sets in $\mathbb{R}^{n+1}$, then for any function $f \in \mathcal{M}_{l}\left(V \cap V^{\prime}\right)$ there exist $f_{1} \in \mathcal{M}_{l}(V), f_{2} \in \mathcal{M}_{l}\left(V^{\prime}\right)$ such that $f(z)=$ $f_{1}(z)-f_{2}(z)$ on $V \cap V^{\prime}$. This result, applied to the pair of open sets $U \backslash \Omega$ and $U^{\prime}$, yields that any $f \in \mathcal{M}_{l}\left(U^{\prime} \backslash \Omega\right)$ can be expressed as $f(z)=f_{1}(z)-f_{2}(z)$ with $f_{1} \in \mathcal{M}_{l}(U \backslash \Omega)$ and $f_{2} \in \mathcal{M}_{l}\left(U^{\prime}\right)$. Then $\rho\left(f_{1}\right)=f$. 
Mittag-Leffler theorem can be written in cohomological terms as:

Theorem 3.5. (Mittag-Leffler) Let $U \subseteq \mathbb{R}^{n+1}$ be an open set. Then

$$
H^{1}\left(U, \mathcal{M}_{l}\right)=0
$$

Remark 3.6. The cohomological formulation of the result allows to write the relative cohomology sequence of the pair $(U, U \backslash \Omega)$ with coefficients in the sheaf $\mathcal{M}_{l}$ which gives

$$
\begin{gathered}
0 \rightarrow H_{\Omega}^{0}\left(U, \mathcal{M}_{l}\right) \rightarrow H^{0}\left(U, \mathcal{M}_{l}\right) \rightarrow H^{0}\left(U \backslash \Omega, \mathcal{M}_{l}\right) \\
\rightarrow H_{\Omega}^{1}\left(U, \mathcal{M}_{l}\right) \rightarrow H^{1}\left(U, \mathcal{M}_{l}\right) \rightarrow \ldots
\end{gathered}
$$

The Mittag-Leffler theorem and the unique continuation property for monogenic functions, see [2], imply $H_{\Omega}^{0}\left(U, \mathcal{M}_{l}\right)=0$ and so we deduce

$$
\mathcal{B}_{l}(\Omega)=\frac{\mathcal{M}_{l}(U \backslash \Omega)}{\mathcal{M}_{l}(U)} \cong H_{\Omega}^{1}\left(U, \mathcal{M}_{l}\right)
$$

Remark 3.7. In alternative, see [17], $\mathcal{B}_{l}(\Omega)$ can be defined as

$$
\mathcal{B}_{l}(\Omega)=\frac{\mathcal{M}_{l}\left(\mathbb{R}^{n+1} \backslash \bar{\Omega}\right)}{\mathcal{M}_{l}\left(\mathbb{R}^{n+1} \backslash \partial \Omega\right)}
$$

Remark 3.8. The set of right monogenic hyperfunctions in $\Omega$ is defined in a similar way: it suffices to change the subscript " $l$ " with " $r$ " in the above definition. It is immediate to verify that $\mathcal{B}_{l}(\Omega)$ is a right $\mathbb{C}_{n}$-module.

We also have the following result, whose proof follows the lines of the analogous result in the complex setting:

Theorem 3.9. The assignment $\Omega \mapsto \mathcal{B}_{l}(\Omega)$ is a flabby sheaf of $\mathbb{C}_{n}$-modules.

Proof. Let $\Omega$ and $\Omega^{\prime}$ be two open sets in $\mathbb{R}^{n}$ and assume that $\Omega^{\prime} \subseteq \Omega$. We show that the restriction map $\mathcal{B}_{l}(\Omega) \rightarrow \mathcal{B}_{l}\left(\Omega^{\prime}\right)$ is surjective. To this end, take $f \in \mathcal{B}_{l}\left(\Omega^{\prime}\right)$, let $\partial \Omega^{\prime}$ be the boundary of $\Omega^{\prime}$ and set $V=\mathbb{R}^{n+1} \backslash \partial \Omega^{\prime}$. The set $\Omega^{\prime}$ is a closed subset of $V$, so $f$ admits a representative $F \in \mathcal{M}_{l}\left(V \backslash \Omega^{\prime}\right)=\mathcal{M}_{l}\left(\mathbb{R}^{n+1} \backslash \overline{\Omega^{\prime}}\right)$. Since $\Omega^{\prime} \subseteq \Omega$, we have that $F \in \mathcal{M}_{l}\left(\mathbb{R}^{n+1} \backslash \bar{\Omega}\right)$. Since $W=\mathbb{R}^{n+1} \backslash \partial \Omega$ is a neighborhood of $\Omega$ we have that $F \in \mathcal{M}_{l}(W \backslash \Omega)$ and $[F]$ defines a hyperfunction $g \in \mathcal{B}_{l}(\Omega)$. Obviously $g_{\mid \Omega^{\prime}}=f$, and the statement follows.

Let now $K \subset \mathbb{R}^{n}$ be a compact set. Then $\mathcal{M}_{l}(K)$ is defined, also topologically, as inductive limit. Its dual module $\mathcal{M}_{l}^{\prime}(K)$ consists of all continuous left $\mathbb{C}_{n}$-analytic functionals on $\mathcal{M}_{l}(K)$. The following result has been proved in [1]:

Theorem 3.10. Let $K \subset \mathbb{R}^{n+1}$ be a compact set. Then

$$
\mathcal{M}_{l}^{\prime}(K) \cong \mathcal{M}_{r}^{0}\left(\mathbb{R}^{n+1} \backslash K\right), \quad \mathcal{M}_{r}^{\prime}(K) \cong \mathcal{M}_{l}^{0}\left(\mathbb{R}^{n+1} \backslash K\right),
$$

where $\mathcal{M}_{r}^{0}\left(\mathbb{R}^{n+1} \backslash K\right)$ (resp. $\mathcal{M}_{l}^{0}\left(\mathbb{R}^{n+1} \backslash K\right)$ ) denotes the set of right (resp. left) monogenic functions outside $K$ which vanish at infinity. 
One may use the classical definition of hyperfunctions in $\Omega \subseteq \mathbb{R}^{n}$ in order to construct hyperfunctions with values in $\mathbb{C}_{n}$. It can be done as follows. Let us consider functions of the form $F=\sum_{A} F_{A} e_{A}$ where $F_{A} \in \mathcal{B}(\Omega)$. The set of such functions endowed with a left multiplication by an element in $\mathbb{C}_{n}$ will be denoted by $\mathcal{B}_{(l)}(\Omega)$ and, similarly, when it is endowed with a multiplication on the right by an element in $\mathbb{C}_{n}$ it will be denoted by $\mathcal{B}_{(r)}(\Omega)$. Then $\mathcal{B}_{(l)}(\Omega)$ (resp. $\mathcal{B}_{(r)}(\Omega)$ ) is called the left (resp. right) $\mathbb{C}_{n^{-}}$-module of $\mathbb{C}_{n^{-}}$ valued hyperfunctions on $\Omega$. The following result has been proved in [27] but we briefly sketch the proof for the sake of completeness:

Theorem 3.11. There are the following $\mathbb{C}_{n}$-module isomorphisms:

$$
\mathcal{B}_{l}(\Omega) \cong \mathcal{B}_{(r)}(\Omega) \quad \text { and } \quad \mathcal{B}_{r}(\Omega) \cong \mathcal{B}_{(l)}(\Omega)
$$

Proof. Let us denote by $\mathcal{A}$ the sheaf of real analytic functions with values in $\mathbb{C}_{n}$; then consider the left $\mathbb{C}_{n}$-module $\mathcal{A}_{(l)}(K)$ of real analytic $\mathbb{C}_{n}$-valued functions where $K$ is a compact subset of $\mathbb{R}^{n}$. It can be shown, by the uniqueness of the CK-extension, see [27], that since $K \subset \mathbb{R}^{n}$

$$
\mathcal{A}_{(l)}(K)=\mathcal{M}_{r}(K) \text {. }
$$

On the other hand, these $\mathbb{C}_{n}$-modules have to be interpreted as inductive limits, and so

$$
\mathcal{A}_{(l)}(K)=\mathcal{M}_{r}(K)=\text { ind } \lim \mathcal{M}_{r}(K+B(0, \varepsilon))
$$

where $K+B(0, \varepsilon)=\left\{x_{0}+\underline{x} \in \mathbb{R}^{n+1} \mid x_{0}+\underline{x}=\underline{y}+z, \underline{y} \in \mathbb{R}^{n}, z \in B(0, \varepsilon)\right\}$. Theorem 3.10 gives

$$
\mathcal{A}_{(l)}^{\prime}(K)=\mathcal{M}_{r}^{\prime}(K) \cong \mathcal{M}_{l}^{0}\left(\mathbb{R}^{n+1} \backslash K\right) .
$$

Let us assume that $\Omega$ is relatively compact in $\mathbb{R}^{n}$. Then, using (3) and a well known result proved by Martineau, in [17] we immediately have

$$
\mathcal{B}_{l}(\Omega)=\frac{\mathcal{A}_{(l)}^{\prime}(\bar{\Omega})}{\mathcal{A}_{(l)}^{\prime}(\partial \Omega)} \cong \mathcal{B}_{(r)}(\Omega) .
$$

Assume now that $\Omega$ is any open set in $\mathbb{R}^{n}$ and let $f \in \mathcal{B}_{l}(\Omega)$. By virtue of the previous isomorphism, for any $\Omega^{\prime} \subset \Omega$ relatively compact, there exists a unique $g^{\prime} \in \mathcal{B}_{(r)}\left(\Omega^{\prime}\right)$ which corresponds to $f_{\mid \Omega^{\prime}}$. If $\Omega^{\prime \prime}$ is another relatively compact set in $\Omega$ we can repeat the reasoning and we find $g^{\prime \prime}$ which is such $g_{\mid \Omega^{\prime} \cap \Omega^{\prime \prime}}^{\prime}=g_{\mid \Omega^{\prime} \cap \Omega^{\prime \prime}}^{\prime \prime}$. Since $\mathcal{B}_{l}$ is a sheaf, there exists a unique $g \in \mathcal{B}_{(r)}(\Omega)$ such that $g_{\mid \Omega^{\prime}}=g^{\prime}$ and $g_{\mid \Omega^{\prime \prime}}=g^{\prime \prime}$. The converse is true since for any $g \in \mathcal{B}_{(r)}\left(\Omega^{\prime}\right)$ there exists a corresponding $f^{\prime} \in \mathcal{B}_{l}\left(\Omega^{\prime}\right)$ which extends to $\Omega$ by virtue of the flabbiness of the sheaf $\mathcal{B}_{l}$. As a consequence we get the statement.

From the isomorphism we have just proved, it follows that any monogenic hyperfunction can be seen as a sum of (Sato) hyperfunctions and conversely. However, the approach of Clifford analysis is more effective, as the following examples show, but see also the recent [5]. 
Example 3.12. The Dirac delta can be seen as a hyperfunction at 0 as

$$
\delta(\underline{x})=\left[\frac{2}{A_{n+1}} \frac{\bar{x}}{|x|^{n+1}}\right]
$$

$A_{n+1}$ being the surface area of the sphere of unit 1 -vectors in $\mathbb{R}^{n+1}$. The hyperfunctions $\delta^{ \pm}$which are defined as follows

$$
\begin{aligned}
& \delta^{+}=\left\{\begin{array}{cc}
\frac{2}{A_{n+1}} \frac{\bar{x}}{|x|^{n+1}} & \operatorname{Re}(x)>0 \\
0 & \operatorname{Re}(x)<0
\end{array}\right. \\
& \delta^{-}=\left\{\begin{array}{cc}
0 & \operatorname{Re}(x)>0 \\
\frac{2}{A_{n+1}} \frac{\bar{x}}{|x|^{n+1}} & \operatorname{Re}(x)<0
\end{array}\right.
\end{aligned}
$$

are such that $\delta=\delta^{+}+\delta^{-}$. As we showed in [5], the dirac delta can be represented by taking the scalar part of the restriction of the Cauchy kernel to a parabola, up to a factor, namely if we set $x_{0}=a|\underline{x}|^{2}$, where $x_{0}=\operatorname{Re}(x)$, then we have the new formula

$$
\delta(\underline{x})=\frac{2}{(2 \pi)^{m}}\left[\frac{a|\underline{x}|^{2}-\underline{x}}{\left.|a| \underline{x}\right|^{2}+\left.\underline{x}\right|^{m+1}}(1-a \underline{x})\right]_{0} .
$$

Example 3.13. We now give another example of hyperfunction which naturally arises in Clifford analysis. Let us set

$$
\Lambda_{k}^{ \pm}(f)(x)= \pm \frac{1}{A_{n+1}} \int_{0}^{\infty} \frac{\bar{x} \pm t}{|x \pm t|^{m+2 k+1}} f(t) d t .
$$

We define some hyperfunctions on $\mathbb{R}^{n}$ with singularity at the origin which naturally arises in Clifford analysis. They correspond to a refinement of the classical Riesz potential which may thus be interpreted as a monogenic hyperfunction. Let $P_{k}(\underline{x})$ be an inner spherical monogenic of degree $k$ in $\mathbb{R}^{n}$. Then $\Lambda_{k}^{ \pm}(x) P_{k}(\underline{x})$ is monogenic in $\mathbb{R}^{n+1} \backslash\left\{x \mid \underline{x}=0, x_{0}>\right.$ 0 or $\left.x_{0}<0\right\}$. Hence we can define

$$
\lambda_{k}^{+}(f)(\underline{x})=\Lambda_{k}^{+}(f)(\underline{x}+0) \quad \lambda_{k}^{-}(f)(\underline{x})=\Lambda_{k}^{-}(f)(\underline{x}-0) .
$$

and the hyperfunctions $\lambda_{k}(f)=\lambda_{k}^{+}(f)+\lambda_{k}^{-}(f)$.

Take $f=\chi_{\mathbb{R}_{+}} t^{\alpha}$, where $\chi_{\mathbb{R}_{+}}$is the characteristic function on $\mathbb{R}^{+},-1<\operatorname{Re}(\alpha)<m+2 k+1$ and set $\lambda_{\alpha, k}^{ \pm}=\lambda_{\alpha, k}^{ \pm}\left(\chi_{\mathbb{R}_{+}}\right)$

$$
\begin{aligned}
\lambda_{\alpha, k}^{ \pm}(x) & = \pm \frac{1}{A_{n+1}} \int_{0}^{\infty} \frac{t^{\alpha+1} \mp \underline{x} t^{\alpha}}{\left(|\underline{x}|^{2}+t^{2}\right)^{(n+2 k+1) / 2}} f(t) d t \\
& =\frac{\rho^{\alpha-n+1-2 k}}{A_{n+1}}\left[ \pm \int_{0}^{\infty} \frac{t^{\alpha+1}}{\left(\sqrt{1+t^{2}}\right)^{n+1+2 k}} d t-\underline{\xi} \int_{0}^{\infty} \frac{t^{\alpha}}{\left(\sqrt{1+t^{2}}\right)^{n+1+2 k}} d t\right] \\
& =\rho^{\alpha-n+1-2 k}\left[ \pm \sigma_{m, k}(\alpha+1)-\underline{\xi}(\alpha)\right]
\end{aligned}
$$

where $\rho=|\underline{x}|, \underline{\xi}=\underline{x} /|\underline{x}|$ and

$$
\sigma_{n, k}(\alpha)=\frac{1}{2 A_{n+1}} B\left(\frac{n+2 k-\alpha}{2}, \frac{\alpha+1}{2}\right)=\sigma_{n, k}(n+2 k-1-\alpha) .
$$


Moreover

$$
\lambda_{\alpha, k}(\underline{x}) P_{k}(\underline{x})=-2 \sigma_{n, k}(\alpha) \underline{x}|\underline{x}|^{\alpha-n-k} P_{k}(\underline{\xi}) .
$$

The (left) Hilbert-Riesz transform $\mathcal{H}_{l}$ acts on $\lambda_{k}^{ \pm}(\underline{x}) P_{k}(\underline{x})$ as

$$
\mathcal{H}_{l}\left(\lambda_{\alpha, k}(\underline{x}) P_{k}(\underline{x})\right)=2 \sigma_{m, k}(\alpha+1)|\underline{x}|^{\alpha-m+1-k} P_{k}(\underline{\xi})
$$

which, for $k=0, P_{k}=1$ is the classical Riesz potential.

\section{Avenues for further research}

There is another possibility to look at hyperfunctions supported in $\mathbb{R}^{n}$, in fact one may also construct hypefunctions with values in the bicomplex numbers, see [6], [7]. These are constructed as $n$-relative cohomology class with values in the sheaf of bicomplex holomorphic functions.

In the three dimensional case, we have another possibility to consider classes of functions whose boundary values give hyperfunctions supported in $\mathbb{R}^{3}$. Indeed, in we can also consider boundary values of Cauchy-Fueter regular functions on $\tilde{\mathbb{H}}=\{q \in \mathbb{H} \mid \operatorname{Re}(q)=0\}$, see [10], [19], [23]. In the case of Cauchy-Fueter regular functions, one may also wonder to extend the theory to the case of of hyperfunctions in several variables and with values in the quaternions. The theory is completely described in the case of two variables, see [3]. Here the main problem is to understand what is the variety on which the sheaf of hyperfunctions is supported. To this end it will be necessary to prove the quaternionic analogue of the pure $n$-codimensionaliy of $\mathbb{R}^{n}$ in $\mathbb{C}^{n}$.

In [4] we have discussed how to generalize the notion of monogenic hyperfunctions to the case of several variables. As it happens in the case of Sato's hyperfunctions, the definition should be a cohomological one. We have shown that the problem is intimately related with the problem of finding a minimal free resolution for the module generated by the rows of the matrix associated to the symbol of several Dirac operators. This problem has been treated originally in [21] and, from another point of view, in [20]. We conjectured that when we consider monogenic functions with values in $\mathbb{C}_{n}$ and we consider $k$ operators, if $n \geq 2 k-1$ then the resolution can be obtained using the radial algebra relations. In [21] we have proved that an alternative hypothesis which guarantees that the resolution is radial is to assume that the first syzygies are at most quadratic. So far, the general statement has not been proved. It will be interesting to use commutative algebra methods, maybe combined with the theory of invariant operators, to prove the result and obtain the description of monogenic hyperfunctions in several variables.

Finally, we mention that in [9] and related papers the theory of monogenic functions has been extended to a superspace. By using similar techniques as in the present paper, one may thus arrive at a natural extension of the theory of hyperfunctions to superanalysis.

\section{References}

[1] R. Delanghe, F. Brackx, Duality in hypercomplex function theory, J. Funct. Anal. 37 (1980), 164-181. 
[2] F. Brackx, R. Delanghe, F. Sommen, Clifford Analysis, Pitman Res. Notes in Math., $76,1982$.

[3] F. Colombo, A. Damiano, I. Sabadini D.C. Struppa, Quaternionic hyperfunctions on 5-dimensional varieties in $\mathbb{H}^{2}$, Journal of Geometric Analysis, 17 (2007), 459-478.

[4] F. Colombo, I. Sabadini, F. Sommen, D.C. Struppa, Analysis of Dirac Systems and Computational Algebra, Progress in Mathematical Physics, Vol. 39, Birkhäuser, Boston, 2004.

[5] F. Colombo, I. Sabadini, F. Sommen, D.C. Struppa, Twisted plane wave expansions using hypercomplex methods, Publ. RIMS Kyoto Univ., 50 (2014), 1-18.

[6] F. Colombo, I. Sabadini, D.C. Struppa, A. Vajiac, M. Vajiac, Bicomplex hyperfunctions, Annali di Matematica Pura ed Applicata, 190 (2011), 247-261.

[7] F. Colombo, I. Sabadini, D.C. Struppa, A. Vajiac, M. Vajiac, Singularities of functions of one and several bicomplex variables, Arkiv Matematik (Institut Mittag Leffler), 49 (2011), 277-294.

[8] R. Delanghe, F. Sommen, V. Souček, Clifford Algebra and Spinor-valued Functions, Mathematics and Its Applications 53, Kluwer Academic Publishers, 1992.

[9] H. De Bie, F. Sommen, Clifford analysis approach to superspace, Annals of Phys., 322 (2007), 2978-2993.

[10] A. Fabiano, G. Gentili, D. C. Struppa, Sheaves of quaternionic hyperfunctions and microfunctions, Compl. Var., 24 (1994), 161-184.

[11] K. Gürlebeck, W. Sprössig, Quaternionic analysis and elliptic boundary value problems, International Series of Numerical Mathematics, 89, Birkhäuser Verlag, Basel, 1990.

[12] K. Gürlebeck, K. Habetha, W. Sprößig, Holomorphic Functions in the Plane and n-dimensional space, Birkhäuser, Basel, 2008.

[13] W. Sprößig, K. Gürlebeck, Clifford analysis and boundary value problems of partial differential equations, Textos de Matemática. Série B, 26, Universidade de Coimbra, Departamento de Matemática, Coimbra, 2000.

[14] A. Kaneko, Introduction to Hyperfunctions, Mathematics and its Applications, Kluwer, 1988.

[15] G. Kato, D. C. Struppa, Fundamentals of Algebraic Microlocal Analysis, Marcel Dekker Inc., New York, 1999.

[16] G. Köthe, Die Randverteilungen analytischer Funktionen, Math. Zeit. 57 (1952), 1333.

[17] A. Martineau, Les hyperfonctions de M. Sato, Séminaire Bourbaki, 214, (1960). 
[18] M. Morimoto, An Introduction to Sato's Hyperfunctions, Trans. Math. Monographs, Vol. 129 A.M.S., Providence, Rhode Island, 1993.

[19] I. Sabadini, Verso una teoria delle iperfunzioni quaternioniche, PhD dissertation, Milano 1995.

[20] I. Sabadini, F. Sommen, D. C. Struppa, The Dirac complex on abstract vector variables: megaforms, Exp. Math., 12 (2003), 351-364.

[21] I. Sabadini, F. Sommen, D. C. Struppa, P. Van Lancker, Complexes of Dirac operators in Clifford algebras, Math. Zeit., 239 (2002), 215-240.

[22] M. Sato, Theory of hyperfunctions, Sūgaku, 10 (1958), 1-27.

[23] I. Sabadini, D. C. Struppa, Topologies on quaternionic hyperfunctions and duality theorems, Compl. Var., 30 (1996), 19-34.

[24] M. Sato, T. Kawai, M. Kashiwara, Microfunctions and Pseudo-Differential Equations, Springer LNM, Vol. 287 (1973), 265-529.

[25] M. Sato, Theory of hyperfunctions, J. Fac. Sci. Univ. Tokyo, Sect. I., 8 (1958), 139-193 and 387-436.

[26] L. Schwartz, Théorie des Distributions I, II, Hermann, Paris, 1950-51.

[27] F. Sommen, Hyperfunctions with values in a Clifford algebra, Simon Stevin, Quart. J. Pur Appl. Math. 57 (1983), 225-254.

[28] F. Sommen, Microfunctions with values in a Clifford algebra, II, Sci. Papers College of Arts and Sciences, Univ. Tokyo, 36 (1986), 15-37. 